\title{
NOTAS
}

\section{LA MEDIA SUBIDA DE CLÍTICOS EN EL ESPAÑOL DE HOUSTON}

\section{INTRODUCCIÓN}

Un aspecto relacionado con los clíticos en español, que ha llamado la atención de los especialistas, es la "subida", fenómeno que la gramática del español permite como resultado de una reestructuración de complementos oracionales que pasan a formar parte de una oración mayor, en el contexto de ciertas perífrasis constituidas por un verbo en infinitivo o gerundio y un verbo conjugado que funciona a modo de auxiliar. El proceso mencionado se observa en los ejemplos siguientes: en ( $1 a$ ) el clítico se encuentra adherido a la forma no finita; en (1b), éste "ha subido" a la oración mayor ubicándose antes del verbo conjugado:

(1) a. puedo comprarlo $\rightarrow$ b. lo puedo comprar

Una combinación de dos verbos no finitos también puede aceptar una subida de los clíticos cuando aquéllos conforman una unidad. Como se ilustra en (2)-(4), las versiones (2a), (3a) y (4a) llevan añadido el clítico al verbo que aporta el significado léxico a la frase; en (2b), (3b) y (4b), en cambio, el clítico ha subido, aunque "a medias", hacia la forma no finita que se encuentra en una posición más elevada:

(2) $a$. ir a verlo $\rightarrow b$. irlo a ver

(3) $a$. volver a hacerlo $\rightarrow b$. volverlo a hacer

(4) $a$. tratar de sacarla $\rightarrow b$. tratarla de sacar

En frases verbales del tipo (2)-(4), el clítico puede ubicarse también antes del verbo conjugado, si lo hay, posición que sólo es posible actualmente cuando el verbo es finito. Este contexto se produce a raíz de la función de complemento que pueden cumplir los verbos 
no finitos ("quisiera poder decirle", "voy a ir a visitarlos"), a raíz de su valor nominal ("el poder detenerlo") o a la función adverbial del gerundio ("vas a estar aburriéndote").

El presente trabajo intenta determinar las preferencias de los hablantes por la media subida de clíticos en el español hablado en Houston. En particular, los datos provienen de la variedad de español que se encuentra en contacto con el inglés, variedad utilizada por los hablantes méxico-americanos que residen en esta ciudad.

En la jerarquía de complejidad de las lenguas, este tipo de alternancia parece encontrarse en los niveles más elevados; así se podría explicar la dificultad para determinar la aparente o real sinonimia de las construcciones correspondientes. La construcción estudiada, desde el punto de vista referencial, ofrece dos formas que permiten al hablante aportar un mismo significado. Cuando el hablante dispone de una sola forma para expresar un determinado significado, la selección se produce de manera automática de acuerdo con la exigencia del contexto lingüístico específico; cuando dispone de dos formas, sin embargo, la selección podría estar determinada por matices de significado difíciles de establecer, no sólo para el hablante común, sino también, en muchos casos, para el especialista. Los contextos lingüísticos con estas características presentan límites difusos, por lo que los hablantes tienden a interpretar las formas disponibles como sinónimas. Esta identificación podría ser un paso más hacia la desaparición de una de las formas en competencia; si éste fuera el caso, habría que determinar cuál es la forma favorecida por los hablantes.

Hay contextos lingüísticos en que la alternancia se verifica entre formas que tienen sus significados claramente delimitados, por lo tanto, lo que se observa en ellos no es realmente una alternancia libre. Casos como "ser" vs. "estar", "por" vs. "para", "imperfecto" vs. "pretérito" e "indicativo" vs. "subjuntivo" son oposiciones que ilustran un tipo de opción en que la selección de una u otra forma de la oposición se encuentra determinada por el contexto sintácticosemántico en que aparece. Cuando "ser" y "estar" se construyen con predicados de naturaleza adjetival, por ejemplo, la selección de estas formas se encuentra determinada por restricciones semánticas del tipo 'marco de clase' vs. 'marco individual', distinciones que el hablante nativo utiliza para determinar cuál de las dos formas es la esperable en una construcción dada. En algunas variedades del español, sin embargo, cierto grado de neutralización entre estas formas ha ocurrido en el contexto mencionado, ya que "estar" se usa por los hablantes para expresar también marco de clase, significado tradicionalmente expresado con la forma "ser"1. Esta extensión

1 Manuel J. Gutiérrez, "Ser"y “estar” en el habla de Michoacán, UNAM, México, 1994. 
semántica de "estar" ha añadido mayor complejidad a la oposición, ya que las formas han comenzado a competir en los mismos contextos y los hablantes las perciben como sinónimas. Otro ejemplo de contextos que se complican debido a la neutralización que ocurre entre dos o más formas que compiten por los mismos espacios semánticos lo constituye la desaparición del futuro de subjuntivo en español, proceso que fue precedido por la identificación de las formas de este tiempo verbal con las del pretérito imperfecto de subjuntivo.

La complejidad a la que se enfrentan los hablantes radica, entonces, en las opciones que tienen a su alcance, y en la identificación del elemento al que unen el clítico. Se podría pensar que la cercanía del clítico al verbo que funciona a modo de auxiliar da un carácter más abstracto a la construcción y que, por lo tanto, ésta tendría una complejidad mayor a su equivalente sin subida de clítico. Su ligazón al verbo principal, por otro lado, ofrece más transparencia a la construcción, pues ayuda a poner acento en el significado léxico de ésta. También es cierto, sin embargo, que el clítico, en este último caso, se encuentra en una posición más incrustada, lo cual conlleva un proceso mayor, tanto en su elaboración como en su decodificación. Se podría argumentar que la anteposición permite poner en una posición central al clítico y su referente, lo que haría menos compleja la construcción que su opuesta. En construcciones de este tipo, habría que considerar, sin embargo, una variedad de factores, entre ellos el tipo de verbo que funciona como auxiliar, cuestión que parece ser fundamental en el fenómeno de subida de clíticos. Para determinar la complejidad de una estructura, lo más apropiado es estudiar el desempeño de los hablantes al producir las distintas combinaciones, en especial aquellas en las que pueden optar por más de una de ellas. Las preferencias de los hablantes y las características de éstos pueden aportar información valiosa al respecto.

\section{Metodología}

El presente trabajo estudia las estructuras mencionadas compuestas de verbos no finitos con datos actuales del español hablado en Houston. Noventa y seis horas de grabación con el mismo número de hablantes méxico-americanos fueron examinadas con el propósito de extraer las frases verbales que permiten la subida del clítico. La muestra de hablantes consiste en 53 mujeres y 43 hombres, y se encuentra distribuida en tres generaciones definidas según la procedencia de los padres, el lugar de nacimiento o llegada a los Estados Unidos y el número de años que han estado en contacto con el inglés. De un total de 3196 construcciones que permiten la subida de clíticos, se 
seleccionaron aquéllas consistentes en construcciones con dos verbos no conjugados. Luego, se procedió a establecer correlaciones entre la variable dependiente, posición del clítico, y las variables independientes consideradas: generación y tipo de verbo auxiliar.

Una de las características de las estructuras complejas, especialmente si éstas son de naturaleza gramatical, es su baja frecuencia en el discurso natural de los hablantes; esto y la dificultad para determinar la sinonimia complican su estudio ${ }^{2}$. En el caso del presente trabajo se encontraron 159 casos que calzaban dentro de la definición presentada previamente. Por esta razón, y con el propósito de complementar los datos de las entrevistas, se utilizó un cuestionario especialmente preparado para elicitar las preferencias de los hablantes en cuanto a la posición del clítico en estas estructuras. El instrumento estuvo compuesto de diecinueve oraciones, de las cuales trece eran oraciones de prueba; las seis restantes sólo intentaban evitar la sistematización de respuestas por parte de los hablantes. 71 cuestionarios aplicados a estudiantes universitarios de distintos niveles de la carrera de español permitieron construir una segunda base de datos para establecer nuevas correlaciones entre la posición del clítico, el verbo auxiliar de la construcción, y la primera y segunda lenguas de los hablantes.

Los datos extraídos de ambos instrumentos se codificaron de acuerdo con las variables lingüísticas y sociales mencionadas, y el Paquete Estadístico para las Ciencias Sociales (SPSS) se utilizó para hacer los análisis cuantitativos.

\section{RESUltados}

Un total de 159 clíticos producidos en el corpus oral fueron codificados de acuerdo con las variables lingüísticas y sociales investigadas. De éstos, 124 se encontraban en contextos de frases con verbos no conjugados; los restantes contextos presentaban además la posibilidad de la subida completa del clítico hasta antes de la forma verbal conjugada. En el primer caso, sólo las dos posiciones del español actual eran posibles; en el último, tres. La Tabla 1 muestra los porcentajes de aparición en la primera situación.

Las frecuencias encontradas indican que la norma de la comunidad muestra que las preferencias se sitúan claramente en la posición

2 Beatriz Lavandera, "Where does the sociolinguistic variable stop?", LangS, 7 (1978), 171-183; Gillian SAN KOFF, "Above and beyond phonology in variable rules", en New ways of analyzing variation in English, eds. C.-J. N. Bailey \& R. Shuy, Georgetown University Press, Washington, D.C., 1973, pp. 42-62; Carmen Silva-Corvalán, Sociolingüistica: teoría y análisis, Alhambra, Madrid, 1989, pp. 97-100. 
TABLA 1

Posición del clítico y generación (oral)

\begin{tabular}{|l|c|c|r|r|}
\hline & \multicolumn{4}{|c|}{ Generación } \\
\hline Posición & Primera & Segunda & Tercera & Total \\
\hline Vbo. -conj. 2_- & $58 \%(29)$ & $89 \%(32)$ & $97 \%(37)$ & $79 \% \quad(98)$ \\
\hline Vbo. -conj. 1_ & $42 \%(21)$ & $11 \%(4)$ & $3 \%(1)$ & $21 \% \quad(26)$ \\
\hline Total & $100 \%(50)$ & $100 \%(36)$ & $100 \%(38)$ & $100 \%(124)$ \\
\hline
\end{tabular}

$\left(\mathrm{X}^{2}=23.167, \mathrm{p} .000\right)$

posterior al segundo verbo no conjugado de la construcción, como en los ejemplos (5-7), indicada por una frecuencia de 79\%, mientras la posición posterior al primer verbo no conjugado, como en los ejemplos (8-9), alcanza una frecuencia de $21 \%$ :

(5) Es bueno saber un poco antes sobre la historia, antes de ir a verla, pero es una buena película $(\mathrm{H}, \mathrm{G} 2)^{3}$.

(6) Mi mamá iba a clases de inglés para poder entendernos a nosotros (M, G2).

(7) ...no tienen la culpa de que sus padres dejaran su país para venir a mejorarse aquí en Estados Unidos (M, G3).

(8) ...muchos no se aguantaban ahí, no se esperaban a que salieran para irnos a bañar al río (H, G1).

(9) Yo digo, yo no estoy para estarme metiendo en eso (M, G1).

Lo más interesante se observa, sin embargo, en las diferencias que presentan los grupos separados de acuerdo con la variable generación. En este caso, se aprecia claramente que sólo es la primera generación la que mantiene la semi subida con insistencia; este proceso disminuye de manera notable de $42 \%$ a $11 \%$ al pasar de la primera a la segunda generación, y más aún, a sólo 3\% en la tercera generación. Si las posibilidades de poner el clítico aumentan a tres, una vez que se añaden los contextos en que además de las otras dos posiciones el clítico puede subir totalmente hasta antes del verbo no conjugado, la situación se altera un poco, pero no de manera drástica. La Tabla 2 presenta la situación recién descrita:

${ }^{3}$ La información que aparece entre paréntesis al final de algunos ejemplos hace referencia al sexo de los hablantes (H: hombre; M: mujer) y a la generación a la que pertenecen (G1: primera generación, G2: segunda generación y G3: tercera generación). 
TABLA 2

Posición del clítico y generación (con subida completa; oral)

\begin{tabular}{|l|c|c|c|c|}
\hline & \multicolumn{4}{|c|}{ Generación } \\
\hline Posición & Primera & Segunda & Tercera & \multicolumn{2}{|c|}{ Total } \\
\hline _Vbo. + conj. & $13.8 \%(8)$ & $25 \%(12)$ & $28.3 \%(15)$ & $22 \% \quad(35)$ \\
\hline Vbo. - conj. $2-$ & $50 \%(29)$ & $66.7 \%(32)$ & $69.8 \%(37)$ & $61.6 \% \quad(98)$ \\
\hline Vbo. -conj. 1_ & $36.2 \%(21)$ & $8.3 \%(4)$ & $1.9 \% \quad(1)$ & $16.4 \% \quad(26)$ \\
\hline Total & $100 \%(58)$ & $100 \%(48)$ & $100 \%(53)$ & $100 \%(159)$ \\
\hline
\end{tabular}

$\left(\mathrm{X}^{2}=27.625, \mathrm{p} .000\right)$

La adición de la opción preverbal produce una ligera baja en las otras dos posibilidades con verbos no conjugados en la primera generación y algo similar ocurre en la tercera generación. En la segunda sólo baja la frecuencia de la posición posterior al primer verbo no conjugado, pues la posterior al segundo verbo no conjugado se mantiene igual. La tercera posición atrae porcentajes de uso que no cambian el panorama de manera radical en este tipo de estructuras. Este resultado es interesante, ya que, por el contrario, los contextos en que desaparece uno de los verbos no conjugados benefician de manera clara la subida de los clíticos. Gutiérrez ${ }^{4}$ encuentra porcentajes de subida de clíticos de $69.6 \%, 71.6 \%$ y $72.1 \%$ a lo largo de estas tres generaciones cuando hay un verbo conjugado y sólo un verbo no conjugado; otros estudios también encontraron porcentajes altos de subida en el contexto señalado ${ }^{5}$. En el caso de frases con dos verbos no conjugados, los porcentajes que ilustran el mantenimiento del clítico pegado al verbo léxico son bastante altos en comparación con los contextos en que sólo hay un verbo no conjugado. Podría afirmarse, por lo tanto, que la adición de un segundo verbo no conjugado agrega también algún factor que hace la subida y media subida de clíticos menos probable ${ }^{6}$.

${ }^{4}$ Manuel J. Gutiérrez, "Restringiendo la subida de clíticos: reflexividad, modalidad verbal y contacto lingüístico en el español de Houston", Hispanic Research Journal, 9 (2008), núm. 4, 299-313.

${ }^{5}$ John My hill, "Variation in Spanish clitic climbing", en Synchronic and diachronic approaches to linguistic variation and change, ed. T.J. Walsh, Georgetown University Press, Washington, D.C., 1989, pp. 227-250; Carmen Silva-Corvalán y Manuel J. Gutiérrez, "On transfer and simplification: Verbal clitics in Mexican-American Spanish", en Studies in language learning and Spanish linguistics in honor of Tracy D. Terrell, eds. P. Hashemipour, R. Maldonado \& M. van Naerssen, McGraw-Hill, San Francisco, 1995, pp. 302-312; MAR K DAVIES, "Analyzing syntactic variation with computerbased corpora: The case of Modern Spanish clitic climbing”, $H, 78$ (1995), 370-80.

${ }^{6}$ La alta frecuencia encontrada en la posición posterior al segundo verbo no conjugado podría llevar a postular una posible influencia del inglés en esta área de la gramática de los hablantes bilingües y el hecho de que el clítico en inglés aparece 
Lo más interesante de las Tablas 1 y 2, con diferencias estadísticas significativas en su interior según SPSS, es, naturalmente, la tendencia que se aprecia en ambas: la posición posterior al primer verbo no conjugado disminuye de manera drástica. La complejidad de la construcción permite ocupar la posición posterior al primer verbo en $42 \%$ y $36.2 \%$, según esté o no el verbo conjugado, a los hablantes de la primera generación, los cuales poseen una variedad de lengua más parecida a la de los hablantes monolingües. En las generaciones sucesivas, esta posición va desapareciendo de la lengua oral de los hablantes; las posibilidades que ofrece la lengua se reducen en este contexto probablemente debido a la complejidad del mismo. Aún cuando la subida de clíticos tradicional se mantiene vigente en todos los grupos, según los estudios anteriores citados más arriba (véase nota 5), este contexto resulta más complejo para los hablantes de generaciones cuyo dominio de la lengua ha decrecido. Considerando el comportamiento de los hablantes frente a este fenómeno, se podría decir que la posición posterior al primer verbo no conjugado, la media subida, es la de mayor complejidad en esta estructura.

La posición posterior al primer verbo no conjugado parece ser una característica de los hablantes nativos del español. Keniston, en su estudio de varios géneros en prosa de diferentes regiones del mundo hispano entre 1900 y 1930 , encuentra 62\% (99 casos) de clíticos después del primer verbo no conjugado y $38 \%$ (61 casos) de clíticos después del segundo verbo. Este rasgo se mantuvo con insistencia a lo largo del siglo xx. En 100 horas de grabación con un total de 141 hablantes cultos de la Ciudad de México, Luna Traill ${ }^{8}$ encuentra $62 \%$ de clíticos después del primer infinitivo (42 casos: "Tengo la idea de poderlo dar") y $38 \%$ después del segundo infinitivo (26 casos) $)^{9}$.

Resultados de siglos anteriores también confirman la preferencia por esta posición. Keniston, en su estudio de la prosa castellana del siglo $\mathrm{XVI}^{10}$, encuentra $63 \%$ (81 casos) después del primer verbo no

de manera categórica en la posición posverbal justificaría la hipótesis. Sin embargo, las altas frecuencias de la subida completa en las tres generaciones documentadas por GUTIÉRREZ (art. cit.) no permiten sostenerla.

7 Hayward Keniston, Spanish syntax list: A statistical study of grammatical usage in contemporary Spanish prose on the basis of range and frequency, Holt, Rinehart and Winston, New York, 1937.

8 Elizabeth Luna Traill, "Sobre la sintaxis de los pronombres átonos en construcciones de infinitivo", ALM, 10 (1972), 191-200.

${ }^{9}$ La diferencia de porcentajes entre la primera generación de Houston y los hablantes monolingües de la Ciudad de México (E. Luna Traill, art. cit.) indicaría que la exposición a una segunda lengua en una forma permanente podría afectar la media subida, ya que el grupo de Houston presenta un porcentaje menor de media subida que el grupo monolingüe (Luis Ortiz López en conversación personal).

${ }^{10} \mathrm{H}$. Keniston, The syntax of the Castilian prose: The sixteenth century, The University of Chicago Press, Chicago, 1937. 
conjugado y $34 \%$ después del segundo (47). Luna Traill y Parodi, en su estudio con documentos americanos judiciales y de otra naturaleza del siglo XVI ${ }^{11}$, encuentran trece casos de construcciones con dos infinitivos; en nueve de éstas (69\%) los clíticos se encuentran después del primer infinitivo ("Lo sabe más de avello oído decir públicamente"), los cuatro restantes (31\%) aparecen después del segundo.

Los datos de la comunidad estudiada en el presente trabajo indican que estamos en presencia de un cambio lingüístico, bastante avanzado al parecer, hacia la eliminación de una de las posibilidades ofrecidas a los hablantes. La notoria reducción de las opciones que mantienen vigentes los hablantes monolingües de español, y que mantienen de modo similar los hablantes de la primera generación de la comunidad bilingüe, es un caso de simplificación y pérdida que ha sido documentado en numerosas situaciones de contacto lingüístico ${ }^{12}$. En casos como el que se discute, los hablantes tienen la posibilidad de recurrir a formas diferentes para expresar un mismo significado; mientras los hablantes nativos y de primera generación mantienen las formas alternantes para expresar distintos matices estilísticos, los hablantes de generaciones posteriores eliminan la o las que les resultan más complejas o simplemente no las adquieren, ya que en un estadio anterior ha ingresado a su sistema lingüístico una de las formas que pueden utilizar en todos los contextos. Como se verá en las páginas siguientes, los hablantes más cercanos a la variedad monolingüe manifiestan diferencias en su actuación lingüística entre el instrumento oral y el escrito, lo que permite apreciar comportamientos diferentes entre un estilo de menor formalidad y uno más formal. En los hablantes más alejados de la variedad monolingüe, en cambio, no se aprecian diferencias estilísticas.

\section{Cuestionario}

Los resultados obtenidos en el cuestionario aplicado ofrecen evidencia que confirma que el proceso de media subida tiene que ver con la cercanía al dialecto nativo de la lengua, probablemente debido a la gran dificultad que este contexto presenta. El grupo de estudiantes

11 Elizabeth Luna Traill y Claudia Parodi, "Sintaxis de los pronombres átonos en construcciones de infinitivo durante el siglo xvi”, ALM, 12 (1974), 196-204.

12 Nancy Dorian, Language death, University of Pennsylvania Press, Philadelphia, 1981; SUSAN GAL, Language shift: Social determinants of linguistic change in bilingual Austria, Academic, New York, 1979; Raymond Mougeon \& Edouard Beniak, Linguistic consequences of language contact and restriction: The case of French in Ontario, Canada, Clarendon Press, Oxford, 1991; Peter Trudgill, "Creolizations in reverse: Reduction an simplification in the Albanian dialects of Greece", Transactions of the Philological Society, 1976-1977, 32-50. 
universitarios de español fue dividido entre aquellos para quienes ésta es la primera lengua y aquéllos para quienes es la segunda, con el fin de determinar si esta tarea, que requiere de una mayor atención, ofrecía diferencias entre ambos grupos. La Tabla 3 presenta los resultados obtenidos en los que las diferencias son estadísticamente significativas.

TABLA 3

Posición del clítico y grupo (cuestionario)

\begin{tabular}{|l|c|c|c|}
\hline & \multicolumn{3}{|c|}{ Grupo } \\
\hline Posición & Hispanos & -Hispanos & Total \\
\hline Vbo. -conj. 2_ & $76 \%(459)$ & $97 \%(134)$ & $(593)$ \\
\hline Vbo. - conj. 1_ & $24 \%(144)$ & $3 \%(4)$ & $(148)$ \\
\hline Total & $100 \%(603)$ & $100 \%(138)$ & $(741)$ \\
\hline
\end{tabular}

$\left(\mathrm{X}^{2}=39.090, \mathrm{p} .000\right)$

Las preferencias de los hablantes por la media subida están representadas por $24 \%$, lo que indica que el nivel de atención que la tarea requiere favorece menos este proceso que en la lengua oral, en donde llegaba a $42 \%$ en la primera generación. Es interesante notar que el estilo más cuidadoso afecta la frecuencia del fenómeno, disminuyéndolo, mientras el habla más natural lo favorece. Como este contexto es más complejo que la no subida, habría que indicar que el estilo no tiene que ver necesariamente con el nivel de complejidad de las estructuras, sino con la apreciación subjetiva que los hablantes manifiestan cuando toman más tiempo para evaluar las dos variantes alternantes. La comparación de estos resultados con los orales indicaría, por lo tanto, que esta distinción estilística ya no está presente en la tercera generación y que está desapareciendo de manera acelerada en la segunda.

Las diferencias entre los dos grupos considerados en la Tabla 3 indican que la posición posterior al primer verbo no conjugado parece ser exclusiva de quienes adquieren el español como primera lengua, y que quienes la aprenden como segunda pueden alcanzar un dominio muy limitado de ella. Sólo cuatro instancias (3\%) de un total de 138 produjeron los estudiantes de español de último año de licenciatura y de nivel graduado con esta posición. Los estudiantes nativos de español, en cambio, también en estos mismos niveles, produjeron 144 (24\%) de un total de 603, lo que la convierte en una variante activa, como se observó con claridad en los resultados de la lengua oral.

La escasa presencia de alternancia en el grupo que aprendió espanol como segunda lengua indica que la presencia del clítico después del primer verbo no conjugado se aprende tardíamente, si es que se 
aprende en algún momento, ya que este grupo está constituido por hablantes bilingües que han completado el proceso formal de enseñanza de español, hallándose a punto de graduarse en la disciplina o cursando estudios profesionales. Un gran dominio de la lengua en este grupo no garantiza, por lo tanto, el dominio de esta estructura, en particular por encontrarse en los niveles más altos de la jerarquía de complejidad. La estructura examinada presenta un caso en que su aprendizaje constituye la imagen inversa al proceso de pérdida que se verifica en la comunidad bilingüe. La alternancia entre las dos posiciones, y en particular la posición posterior al primer verbo no conjugado, se aprende una vez que se ha logrado un alto dominio de la lengua; la dificultad que ofrece la estructura la convierte en objeto de pérdida temprana en situaciones de bilingüismo intenso y extenso, adquisición de segunda lengua y otras, como ha sido documentado en diversas investigaciones a propósito de otras estructuras ${ }^{13}$.

La observación de los datos permite apreciar la coherencia que hay entre los resultados del cuestionario y los obtenidos en las conversaciones orales. La disminución de la posición posterior al primer verbo, que se aprecia en las generaciones en las Tablas 1 y 2, se relaciona con la edad en que los hablantes adquirieron el español, ya que todos los miembros de la primera generación adquirieron primero esta lengua y luego el inglés. En las generaciones sucesivas, el inglés comienza a ocupar gran parte de las tareas lingüísticas que la sociedad exige de estos hablantes. Esta situación afecta de manera natural su funcionamiento en su primera o segunda lengua, según sea el caso. En el grupo de estudiantes cuya primera lengua es el español, y en aquellos que pertenecen a la primera generación en las conversaciones orales, la posición posterior al primer verbo no conjugado, que se mantenía como una variante activa según los resultados de ambos instrumentos, va desapareciendo a medida que el dominio en la lengua disminuye. Como estructura compleja, en que la alternancia parecería no cumplir un papel semántico y/o pragmático claro, se convierte en una de las primeras víctimas en el habla de quienes no adquirieron la lengua desde el nacimiento, y desaparece de su variedad oral y escrita, pues la variante alternante permite satisfacer sin problemas las necesidades comunicativas en que se usa la estructura. Para los hablantes nativos, sin embargo, ésta es una variante que por siglos ha ido conquistando un espacio y que parece seguir vigente. La media subida es una característica que no tiene la fuerza que ha manifestado a lo largo del tiempo la subida completa, pero es una competidora clara dentro de la variación que se observa en el contexto particular.

13 N. Dorian, op. cit.; C. Silva-Corvalán, Language contact and change: Spanish in Los Angeles, Clarendon Press, Oxford, 1994. 


\section{Auxiliar}

La comparación entre los distintos verbos que aparecen cumpliendo una función de auxiliar en las frases verbales en estudio se hace difícil debido a la gran dispersión que experimentan los clíticos y, por lo tanto, a la reducida frecuencia con que aparecen. Esto es válido tanto en la construcciones con infinitivos como en las construcciones cuyo verbo léxico es un gerundio. La Tabla 4 ofrece los resultados sólo de aquellas formas que mostraron clíticos en la posición posterior al primer verbo no conjugado o en ambas:

TABLA 4

Posición del clítico y verbo auxiliar (oral)

\begin{tabular}{|l|r|r|r|}
\hline & \multicolumn{3}{|c|}{ Posición } \\
\hline Auxiliar & Vbo-conj 2_ & Vbo. -conj 1_ & Total \\
\hline ir & $78 \%(32)$ & $22 \%(9)$ & $(41)$ \\
\hline hacer & & $100 \%(1)$ & $(1)$ \\
\hline poder & $73 \%(22)$ & $27 \%(8)$ & $(30)$ \\
\hline querer & $60 \%(3)$ & $40 \% \quad(2)$ & $(5)$ \\
\hline tener & $92 \%(11)$ & $8 \%(1)$ & $(12)$ \\
\hline necesitar & & $100 \%(1)$ & $(1)$ \\
\hline saber & $67 \%(2)$ & $33 \%(1)$ & $(3)$ \\
\hline volver & & $100 \%(1)$ & $(1)$ \\
\hline seguir + gerundio & $67 \%(2)$ & $33 \%(1)$ & $(3)$ \\
\hline estar + gerundio & $91 \%(10)$ & $9 \%(1)$ & $(11)$ \\
\hline Total & $79 \%(98)$ & $21 \%(26)$ & $(124)$ \\
\hline
\end{tabular}

$\left(\mathrm{X}^{2}=45.545, \mathrm{p} .132\right)$

Los resultados que se observan en la Tabla 4 no son estadísticamente significativos; son de interés, sin embargo, ya que permiten comparar el comportamiento de los hablantes en el contexto de una conversación y frente a un instrumento escrito. La lista de verbos que aparecen cumpliendo alguna función de auxiliar tiene formas que normalmente aceptan la subida tradicional de clíticos; entre éstas están las formas que aparecen acompañadas de gerundios que, como se ha demostrado, expulsan con bastante frecuencia al clítico hacia la posición preverbal ${ }^{14}$. En la media subida, sin embargo, se aprecia con

14 Rena Torres Cacoullos, "Construction frecuency and reductive change: Diachronic and register variation in Spanish clitic climbing”, LVC, 11 (1999), 143170; M. Gutiérrez, op. cit. y "La subida de clíticos durante los siglos XVI-XviII", en Actas del VII Congreso Internacional de Historia de la Lengua Española, eds. C. Company Company y J.G. Moreno de Alba, Arco/Libros, Madrid, 2008, pp. 641-655. 
nitidez que no ocurre lo mismo, ya que la frecuencia de clíticos adheridos a formas de gerundio con auxiliares del tipo "estar" y "seguir" es similar a la que aparece con verbos del tipo "tener", "saber" y otros construidos con infinitivo. "Ir", "poder" y "querer", verbos que aparecen de manera recurrente en la lengua oral, presentan frecuencias de cierta consideración en construcciones con un segundo infinitivo en ambas posiciones y permiten ver la tendencia natural que se ha observado en los resultados más generales de este trabajo. Los clíticos aparecen en nueve de 41 instancias después del primer verbo no conjugado cuando éste es "ir" (22\%), cuando es "poder", ocho de 30 (27\%); cuando el primer infinitivo es "querer", las preferencias se dividen, pero la presencia de los clíticos es más escasa con esta forma, ya que, aunque éstos aparecen con $40 \%$ después de "querer", el número de casos es bajo en ambas posiciones (dos después de este verbo y tres después del segundo infinitivo).

Los resultados obtenidos en el cuestionario ilustran de manera más clara la variación que se produce en este contexto debido al mayor número de casos que se ubica en cada casilla, como se observa en la Tabla 5:

TABLA 5

Posición del clítico y verbo auxiliar (cuestionario; Grupo: Hisp)

\begin{tabular}{|c|c|c|c|}
\hline & \multicolumn{3}{|c|}{ Posición } \\
\hline Auxiliar & Vbo-conj 2 & Vbo. - conj 1_ & Total \\
\hline tratar & $67 \% \quad(37)$ & $33 \% \quad(18)$ & (55) \\
\hline volver & $67 \% \quad(36)$ & $33 \% \quad(18)$ & (54) \\
\hline querer & $70 \% \quad(38)$ & $30 \% \quad(16)$ & (54) \\
\hline ir & $79 \% \quad(67)$ & $21 \% \quad(18)$ & $(85)$ \\
\hline poder & $82 \% \quad(36)$ & $18 \% \quad(8)$ & $(44)$ \\
\hline venir & $85 \% \quad(47)$ & $15 \% \quad(8)$ & $(55)$ \\
\hline tener & $83 \% \quad(68)$ & $17 \% \quad(14)$ & $(82)$ \\
\hline ir + gerundio & $59 \% \quad(32)$ & $41 \% \quad(22)$ & $(54)$ \\
\hline estar + gerundio & $65 \% \quad(34)$ & $35 \% \quad(18)$ & $(52)$ \\
\hline empezar + gerundio & $88 \% \quad(22)$ & $12 \% \quad(3)$ & $(25)$ \\
\hline tratar + gerundio & $98 \% \quad(42)$ & $2 \% \quad(1)$ & (43) \\
\hline Total & $76 \%(459)$ & $24 \%(144)$ & (603) \\
\hline
\end{tabular}

$\left(\mathrm{X}^{2}=1.810, \mathrm{p} .000\right)$

Las perífrasis progresivas son las que más favorecen la media subida con porcentajes de gran proporción, "ir" + gerundio tiene $41 \%$ de casos en esta posición y "estar" + gerundio tiene $35 \%$. Esta prefe- 
rencia está por sobre otras perífrasis que también aceptan la media subida cuando van con gerundios, como las que llevan "empezar" y "tratar"; esto indica que las formas más frecuentes ("ir", "estar"), que señalan movimiento y estado, son también las que más favorecen este fenómeno. Como se dijo anteriormente, la posición preverbal del clítico es casi categórica en este tipo de perífrasis cuando el primer verbo es conjugado, lo que revela un cambio en esta estructura en que la forma innovadora se ha impuesto casi totalmente. En el contexto que permite la media subida, sin embargo, la alternancia sólo puede calificarse en el presente de variación estable cuando se consideran las preferencias de los hablantes hispanos entregadas en el cuestionario. Mayor evidencia de esto se encuentra en la frecuencia que tienen algunos auxiliares construidos con un segundo infinitivo. Tres de ellos, "tratar", "volver" y "querer", ofrecen frecuencias ligeramente más bajas que las recién discutidas: 33\%, 33\% y 30\%, respectivamente. "Ir", "poder", "venir" y "tener" presentan también frecuencias importantes en esta posición, pero por debajo de las anteriores: 21\%, $18 \%, 15 \%$ y $17 \%$, respectivamente. Es difícil determinar las causas que motivan las diferencias que presentan los porcentajes de media subida entre los distintos verbos que cumplen la función de auxiliar en las diferentes perífrasis, pues éstas pueden radicar incluso en las particularidades semánticas de cada una de las construcciones utilizadas en el instrumento.

La comparación entre la producción espontánea y las respuestas al instrumento más estructurado que se utilizó no muestra diferencias importantes al estudiar los distintos auxiliares que aparecen en las construcciones. Éstas aparecían con claridad, sin embargo, cuando comparábamos los resultados generales de media subida en los dos instrumentos, pues se observaba nítidamente que el fenómeno estudiado era favorecido por los hablantes cuando ocupaban la lengua de manera espontánea. Sin embargo, en ninguno de los instrumentos surgen formas que, cumpliendo el papel de auxiliar, se perfilen como indicadoras de alguna tendencia.

Los resultados más generales de media subida y subida de los clíticos ofrecen una diferencia de gran importancia. En el caso del primer fenómeno, el presente estudio concluyó que la situación actual indica una variación estable en el grupo hispano y en la primera generación de hablantes. En la lengua oral, el comportamiento de las distintas generaciones mostró un proceso de cambio tendiente a la eliminación de la opción de la media subida. En este punto se debe señalar que el proceso de subida de clíticos no sólo se conserva vigente en esta comunidad, sino también manifiesta altas frecuencias, lo que la convierte en la variante claramente más utilizada por los usuarios de la lengua. En oposición a los porcentajes de 42\%, 11\%, y 3\%, a través de las generaciones en el caso de la media subida, se encon- 
traron porcentajes de $69.6 \%, 71.6 \%$ y $72.1 \%$, también a lo largo de las generaciones, en el caso de la subida de clíticos ${ }^{15}$. Esta gran diferencia entre los dos fenómenos podría indicar que la subida y media subida de los clíticos son procesos independientes con motivaciones diferentes. Si esto es así, lo más probable es que los hablantes muestren diferencias importantes de subida y media subida también al considerar los auxiliares utilizados. Como se decía más arriba, esto se observa con claridad extrema en el caso de las construcciones de gerundio, pero no es exclusivo de estas construcciones. La Tabla 6, adaptada del artículo de Gutiérrez ${ }^{16}$, ofrece porcentajes de subida (completa) con distintos auxiliares en la comunidad monolingüe de Michoacán y en la comunidad bilingüe de Houston:

TABLA 6

Porcentajes de subida de clíticos con distintos auxiliares

\begin{tabular}{|c|c|c|}
\hline Auxiliar & Michoacán & Houston \\
\hline ir + gerundio & $100 \% \quad(2 / 2)$ & $88.8 \% \quad(16 / 18)$ \\
\hline estar + gerundio & $93.3 \%(14 / 15)$ & $87.6 \%(199 / 227)$ \\
\hline ir a + infinitivo & $83.3 \%(35 / 42)$ & $86.7 \%(359 / 414)$ \\
\hline poder & $81.1 \%(30 / 37)$ & $75.8 \%(217 / 286)$ \\
\hline querer & $76.9 \%(10 / 13)$ & $49.7 \% \quad(94 / 189)$ \\
\hline saber & $75 \% \quad(3 / 4)$ & $26.3 \%$ \\
\hline tratar de & $(1 / 2)$ & $(6 / 19)$ \\
\hline tener que & $28.6 \% \quad(2 / 7)$ & $56.6 \% \quad(90 / 159)$ \\
\hline volver a & & $(9 / 10)$ \\
\hline venir a & & $33.3 \%$ \\
\hline
\end{tabular}

$\left(\mathrm{X}^{2}=293.098, \mathrm{p} .000\right)$

La subida presenta una distribución bastante diferente a la media subida. Los porcentajes de subida muestran una tendencia marcada a favor de la posición anterior al verbo conjugado y, por lo tanto, a su separación del verbo léxico. Esto ocurre tanto en la comunidad bilingüe de Houston como en la monolingüe de Michoacán. En el caso de la media subida, según se observó en los resultados generales por generación, los hablantes nítidamente favorecen de manera creciente, a lo largo de las generaciones, el mantenimiento del clítico junto al verbo léxico, lo que provocará en definitiva la desaparición de la media subida. Esta situación se observa con casi todos los auxiliares.

15 M. GUTIÉRREZ, art. cit.

16 M. Gutiérrez, op. cit. 
Una marcada diferencia entre estos dos procesos se observa en las construcciones con gerundios. En éstas, la subida es casi categórica, pues la frecuencia de aparición en la comunidad que se encuentra en contacto lingüístico presenta porcentajes que están por sobre los 87 puntos, sea "estar" o "ir" el verbo auxiliar, y muy por encima de los 90 puntos en la comunidad monolingüe. La media subida, sin embargo, en el habla espontánea, presenta una frecuencia de casos muy baja y su porcentaje alcanza apenas un tercio de las construcciones. En el caso de los usos menos espontáneos ofrece una frecuencia sólo un poco mayor, pero no supera los 41 puntos cuando se construye con "ir" y ni los 35 cuando se construye con "estar". Con otros auxiliares, los porcentajes son bastante más bajos.

Las diferencias que acabamos de observar no se consumen en las construcciones con gerundio, ya que aquellas con infinitivos señalan también marcadas diferencias en los procesos de subida y media subida. "Volver" e "ir" presentan porcentajes de subida casi tan altos como los vistos en las construcciones con gerundio. Con el primero encontramos nueve casos de diez $(90 \%)$ mientras con "ir" hay un 86.7\% (359/414) en que los clíticos aparecen antes del verbo conjugado. Estos mismos auxiliares, en construcciones de media subida, atraen al clítico con una frecuencia de poco más de 20 o 30\%, ya si consideramos los resultados de los datos orales, ya si los del cuestionario. "Poder" presenta un porcentaje de subida bastante alto en los dos grupos que aparecen en la Tabla 6; en la comunidad bilingüe, aunque tiene un porcentaje inferior al de la monolingüe, se mantiene por sobre los 75 puntos. El porcentaje de media subida sólo alcanza 27\%. "Tener" y "querer" logran atraer porcentajes algo menores de clíticos cuando funcionan a modo de auxiliares; pero de todas maneras le disputan la cercanía del pronombre a los verbos léxicos de las construcciones. En la variedad monolingüe, "querer" favorece claramente la subida (76.9\%) mientras "tener" no logra un gran porcentaje (28.6\%). En el caso de la media subida es poco lo que se puede afirmar en la lengua oral, pero en el cuestionario estas formas no presentan clíticos en grandes proporciones, sólo alcanzan $30 \%$ ("querer") y 17\% ("tener"). En el caso de "tratar" y "venir" se podría decir que existe alguna similitud en ambos procesos en el sentido de que no logran atraer porcentajes importantes de clíticos. En estos casos, tanto en la subida como en la media subida, los porcentajes no son elevados.

Las mayores similitudes entre los dos procesos se encuentran, en realidad, entre aquellos auxiliares que no favorecen (o favorecen menos) ningún tipo de subida. "Querer", que en su función de auxiliar acepta una subida moderada en términos de frecuencia, pues tiene casi $50 \%$, aparece también con frecuencias de cierta proporción en la media subida: 40\% en Houston oral, aunque con pocos 
casos, y 30\% en el cuestionario. "Venir", "tratar" y "saber" son los que menos subida de clíticos aceptan, ya que muestran frecuencias entre 26 y 33\%, lo que es poco en comparación con las formas auxiliares que atraen verdaderamente a los clíticos. La media subida se mueve entre 15 y $33 \%$ con estas mismas formas en función de auxiliar, ya sea en los datos orales, ya en los resultados del cuestionario aplicado. La gran diferencia entre la subida y la media subida es lo más destacable en la comparación entre estos dos procesos; las formas auxiliares que privilegian la subida de los clíticos establecen una distancia marcada con sus equivalentes no conjugadas en las construcciones que permiten la media subida.

\section{ConCLUSIón}

El presente trabajo ha tratado el fenómeno de la media subida de los clíticos, proceso paralelo al de la subida que consiste en el paso del clítico de la última posición, adherido al último verbo no conjugado, al primer verbo sin conjugar. Por el hecho de encontrarse con un verbo no conjugado, el clítico se adhiere a éste y no puede seguir subiendo, a menos que haya además una forma conjugada anterior, caso en el que las opciones para los hablantes aumentan de dos a tres. Los datos examinados del español de Houston, distribuidos según la generación de los hablantes, han dejado en evidencia un proceso de cambio que apunta a la eliminación de la media subida; la disminución marcada y progresiva de clíticos en esta posición sugiere de manera convincente el futuro de esta posición que, de acuerdo con estudios previos, ha gozado de altas preferencias por parte de los hablantes en variedades monolingües del español ${ }^{17}$.

La eliminación de esta posición corresponde a un proceso con características universales que se verifica en todas las situaciones en que los individuos viven con dos lenguas por largos períodos de tiempo. La alta complejidad de algunas formas las hace resistentes a la adquisición o aprendizaje tempranos; por lo mismo, en situaciones de tensión lingüística, son las primeras en desaparecer, especialmente si hay otra forma que se puede expandir para ocupar el espacio semántico dejado por la forma en retirada ${ }^{18}$. La marcada eliminación de la posición posterior al primer verbo no conjugado y la vigencia de este contexto en la primera generación indican que sólo

17 H. Keniston, op. cit.; E. Luna Traill, art. cit.

18 G. Silva-Corvalán, op. cit.; M.J. Gutiérrez, "On the future of the future tense in the Spanish of the Southwest", en Spanish in four continents: Studies in language contact and bilingualism, ed. C. Silva-Corvalán, Georgetown University Press, Washington D.C., 1995, pp. 214-226. 
llega a dominarse después de que el hablante ha alcanzado un alto manejo de la lengua, si es que llega a formar parte alguna vez de su sistema lingüístico. Los hablantes de segunda y tercera generaciones mantienen de manera sostenida la posición posterior al segundo verbo no conjugado, ya que les sirve para expresar los mismos significados que les permite la posición alternante. Para estos hablantes, la diferencia entre las dos formas consideradas por ellos sinónimas ha desaparecido, por lo tanto, mantienen la que les resulta menos compleja.

Los resultados provenientes del cuestionario aplicado a estudiantes de español cuya primera lengua es el español y estudiantes de español cuya primera lengua es el inglés ofrecen mayor evidencia sobre lo recién discutido. Por una parte, la menor frecuencia de formas encontradas en la posición posterior al primer verbo conjugado, comparada con la encontrada para la variedad oral, indica que una situación en que los hablantes prestan mayor atención a la forma favorece la posición posterior al último elemento de la frase verbal. Esto permite afirmar también que la alternancia entre las formas conlleva diferencias pragmáticas, que desaparecerían con la eliminación de una de las posiciones. La comparación de los dos grupos, diferenciados según la lengua que adquirieron primero, muestra que la media subida es un rasgo que puede calificarse de exclusividad nativa, puesto que sólo es parte de quienes han adquirido el español como primera lengua. Los hablantes que lo adquieren como segunda lengua no logran tener un dominio de la media subida que permita considerarla parte de su sistema lingüístico.

En la última parte de este trabajo se intentó explorar las similitudes y diferencias entre los procesos de subida de clíticos y de media subida. Los resultados obtenidos por Gutiérrez (op. cit.) sobre la subida de clíticos con distintas formas que funcionan en este contexto a modo de auxiliar fueron comparados con los resultados encontrados en los dos instrumentos utilizados en la presente investigación. Mientras la subida muestra claros indicios no sólo de mantenerse, sino de ganar terreno en construcciones en que participan varios auxiliares conjugados -tanto en la comunidad bilingüe de Houston como en la comunidad monolingüe de Michoacán-, la media subida parece tener una dirección diferente. La alta complejidad de este proceso lo mantiene con frecuencias bajas y descendentes. No hay comportamientos similares entre los auxiliares que participan en ambos procesos, aun cuando éstos son los mismos, estén conjugados en el caso de la subida o no conjugados en el caso de la media subida. Las mayores similitudes se encontraron en los auxiliares que se comportaron de manera conservadora; es decir, aquellos que no favorecen la subida y media subida de clíticos. 
La baja frecuencia de la estructura estudiada permite llegar sólo a conclusiones tentativas, aunque algunas de éstas parecen claras. Nuevos estudios sobre el tema con mayor número de casos permitirán aportar más evidencia que confirme o rechace los hallazgos del presente trabajo.

MANuel J. GutiérRez

University of Houston 